\title{
Biochemical and transcriptome analyses of a novel chlorophyll-deficient chlorina tea plant cultivar
}

Lu Wang ${ }^{1,2,3}$, Chuan Yue ${ }^{1,3}$, Hongli Cao ${ }^{1,3}$, Yanhua Zhou ${ }^{1,3}$, Jianming Zeng ${ }^{1,2,3}$, Yajun Yang ${ }^{1,2,3^{*}}$ and Xinchao Wang ${ }^{1,2,3^{*}}$

\begin{abstract}
Background: The tea plant (Camellia sinensis (L.) O. Kuntze) is one of the most economically important woody crops. Recently, many leaf color genotypes have been developed during tea plant breeding and have become valuable materials in the processing of green tea. Although the physiological characteristics of some leaf color mutants of tea plants have been partially revealed, little is known about the molecular mechanisms leading to the chlorina phenotype in tea plants.

Results: The yellow-leaf tea cultivar Zhonghuang $2(\mathrm{ZH} 2)$ was selected during tea plant breeding. In comparison with Longjing $43(\llcorner\mathrm{~J} 43)$, a widely planted green tea cultivar, ZH2 exhibited the chlorina phenotype and displayed significantly decreased chlorophyll contents. Transmission electron microscopy analysis revealed that the ultrastructure of the chloroplasts was disrupted, and the grana were poorly stacked in $\mathrm{ZH} 2$. Moreover, the contents of theanine and free amino acids were significantly higher, whereas the contents of carotenoids, catechins and anthocyanin were lower in $\mathrm{ZH} 2$ than in LJ43. Microarray analysis showed that the expression of 259 genes related to amino acid metabolism, photosynthesis and pigment metabolism was significantly altered in ZH2 shoots compared with those of LJ43 plants. Pathway analysis of 4,902 differentially expressed genes identified 24 pathways as being significantly regulated, including cysteine and methionine metabolism, glycine, serine and threonine metabolism, flavonoid biosynthesis, porphyrin and chlorophyll metabolism and carotenoid biosynthesis. Furthermore, a number of differentially expressed genes could be mapped to the theanine biosynthesis, chlorophyll biosynthesis and flavonoid biosynthesis pathways. Changes in the expression of genes involved in these pathways might be responsible for the different phenotype of $\mathrm{ZH} 2$.

Conclusion: A novel chlorophyll-deficient chlorina tea plant cultivar was identified. Biochemical characteristics were analyzed and gene expression profiling was performed using a custom oligonucleotide-based microarray. This study provides further insights into the molecular mechanisms underlying the phenotype of the chlorina cultivar of Camellia sinensis.
\end{abstract}

Keywords: Chlorina, Chlorophyll deficiency, Gene expression, Microarray, Tea plant (Camellia sinensis)

\footnotetext{
*Correspondence: yjyang@tricaas.com; wangxinchao@caas.cn

Equal contributors

${ }^{1}$ Tea Research Institute, Chinese Academy of Agricultural Sciences, Hangzhou

310008, China

${ }^{2}$ National Center for Tea Plant Improvement, Hangzhou 310008, China

Full list of author information is available at the end of the article
}

\section{Biomed Central}

2014 Wang et al.; licensee BioMed Central. This is an Open Access article distributed under the terms of the Creative Commons Attribution License (http://creativecommons.org/licenses/by/4.0), which permits unrestricted use, distribution, and reproduction in any medium, provided the original work is properly credited. The Creative Commons Public Domain Dedication waiver (http://creativecommons.org/publicdomain/zero/1.0/) applies to the data made available in this article, unless otherwise stated. 


\section{Background}

Tea plant (Camellia sinensis (L.) O. Kuntze), which is cultivated for the production of a non-alcoholic beverage, is one of the most economically important woody crops worldwide [1]. There are great genetic variations in tea plant germplasms [2,3]. Many leaf color cultivars have recently been developed during tea plant breeding and have become valuable materials in the processing of green tea. For example, Baiye 1 (Anji Baicha) and Xiaoxueya, two albino tea cultivars grown in China, exhibit white young shoots when the environmental temperature is below $20 \mathrm{C}$ in the early spring $[4,5]$. The development of chloroplasts and the accumulation of chlorophyll $a$ and $b$ are blocked in these two albino cultivars at the albinistic stage [5-7]. In addition to the leaf color changes corresponding to lower chlorophyll contents in Baiye 1 and Xiaoxueya, there are also changes in the leaf chemical composition, which is an important determinant of the sensory quality and healthy effects of tea $[4,8]$. Higher total free amino acid concentrations are detected in the leaves of these two cultivars compared with regular green tea leaves $[4,8]$. The albino tea cultivars have received increased attention and are popular with tea consumers in China because of their unique leaf color and abundant free amino acids.

Among chlorophylls, carotenoids and flavonoids/anthocyanins, which are the three main pigment classes in leaf tissue, chlorophyll and carotenoids are the principal pigments that trap light energy in photosynthetic organisms. It has been demonstrated that the development of chlorotic leaves is related to chlorophyll metabolism and chloroplast development [5,7,9-12]. The biosynthesis of chlorophyll occurs in the grana in chloroplasts. A series of enzymatic steps are involved in chlorophyll biosynthesis, which involve the conversion of glutamate to chlorophyll $a$ and chlorophyll $b$ [13]. The chlorophyll biosynthetic pathway is divided into three main parts: (i) the formation of 5-aminolevulinic acid (ALA), (ii) the formation of protoporphyrin IX from eight molecules of ALA, and (iii) the Mg-protoporphyrin pathway producing chlorophyll [14]. Large numbers of leaf color mutants with chlorophyll deficiencies have been identified in plants, and many efforts have been made to elucidate the regulatory pathways affecting chlorophyll biosynthesis.

It has been reported that a barley mutant lacking chlorophyll $b$ shows a chlorina phenotype due to a lack of enzymes converting chlorophyll $a$ into chlorophyll $b$ [15]. The inbred maize line A661 exhibits a dramatic reduction in its chlorophyll content when grown at a temperature below 15C [16]. The chelation of $\mathrm{Mg}^{2+}$ in protoporphyrin IX to form the $\mathrm{Mg}$-protoporphyrin complex is an important step that is unique to chlorophyll production. The enzyme catalyzing this insertion is known as Mg-chelatase, which is composed of three subunits, ChlD, ChlH and ChlI, and when any one of the subunits is mutated, Mg-chelatase activity is abolished $[14,17,18]$. Chlorina mutants defective in genes encoding the Mg-chelatase subunits have been identified in various plants [14]. The mutants Arabidopsis ch42-3 and gun 5 lack $\mathrm{ChlI}$ and $\mathrm{ChlH}$, respectively, and are deficient in chelating the $\mathrm{Mg}^{2+}$ into protoporphyrin IX $[19,20]$. Rice plants with mutations in OsChlH, OsChlD and OsChlI display the chlorina phenotype [9,11]. Barley mutants lacking $\mathrm{ChlH}, \mathrm{ChlD}$ or $\mathrm{ChlI}$ are unable to synthesize chlorophyll [10]. Either a reduction or excess accumulation of ChlI in tobacco results in the loss of chlorophyll [21]. In addition to genes encoding $\mathrm{Mg}$-chelatase, other genes related to chlorotic leaves have also been identified, such as OsYGL1, which encodes a chlorophyll synthase responsible for catalyzing the esterification of chlorophyllide, OsYLC1, which is a chloroplast-localized gene, and OsLYL1, which encodes a geranylgeranyl reductase [12,22,23]. These genes influence the biosynthesis of chlorophyll and alter chlorophyll contents.

Plants with reduced levels of chlorophyll are the ideal genetic material for exploring the molecular mechanisms regulating the chlorina phenotype and chloroplast development. Compared with studies in model plants with known genome sequences, the lack of genomic information for tea plants has cause molecular biology research in this species to lag behind. Although the physiological characteristics of some leaf color genotypes of tea plants have been partially revealed, little is known about the molecular mechanisms regulating chlorophyll deficiencies in tea plants.

In this study, we compared the biochemical characteristics of two tea cultivars, the normal green tea cultivar Longjing 43 (LJ43) and the novel chlorophyll-deficient chlorina cultivar Zhonghuang 2 (ZH2). The chlorophyll contents of the leaves and the ultrastructure of the chloroplasts of the two cultivars were studied. Furthermore, the levels of carotenoids, theanine, amino acids and flavonoids in the leaves were measured. To reveal the underlying molecular mechanism related to the differences between the two tea cultivars, we used a custom oligonucleotide-based microarray [24] to investigate gene expression in the two cultivars, and identified differentially expressed genes, including genes related to amino acid metabolism, photosynthesis, and pigment metabolism.

\section{Methods}

\section{Plant material and sample preparation}

Two tea plant (Camellia sinensis (L.) O. Kuntze) cultivars, Longjing 43 (LJ43) and Zhonghuang 2 (ZH2), were used in this study. The plants were 5 years old and had been grown in the field at the Tea Research Institute of the Chinese Academy of Agricultural Sciences (TRI,

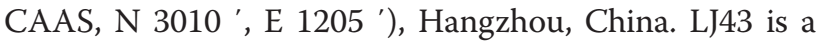


normal green tea cultivar that is widely planted in China, especially in Zhejiang province. ZH2 is a yellow-leaf cultivar that was selected from a natural yellow-leaf mutant in Zhejiang province via systematic selection.

For chemical assays and microarray analysis, the firstly new sprouting fresh shoots (two leaves and one bud) were sampled in spring, and three independent biological replicates were performed. Each replicate was collected from more than ten randomly selected tea plants. The samples were divided into two duplicate, one was steamed for $5 \mathrm{~min}$, dried at $80 \mathrm{C}$ and ground into powder for chemical assay, and the other one was stored at $-80 \mathrm{C}$ after flashfreezing with liquid nitrogen for microarray analysis.

Measurement of chlorophyll, beta-carotene and lutein contents Chlorophyll was extracted with $80 \%$ acetone from $100 \mathrm{mg}$ dried samples. The extract was measured spectrophotometrically at $645 \mathrm{~nm}$ and $663 \mathrm{~nm}$. The chlorophyll content was determined according to the method of Arnon [25], while the lutein and beta-carotene contents were measured via HPLC as described previously [26].

\section{Measurement of theanine, free amino acids, catechins and anthocyanin}

To measure theanine and catechins, $100 \mathrm{mg}$ of the dried samples was extracted at $90 \mathrm{C}$ for $30 \mathrm{~min}$ in $50 \mathrm{ml}$ of water, with shaking once every $10 \mathrm{~min}$. The filtrates were diluted, then refiltered through a $0.45 \mu \mathrm{m}$ nylon filter and analyzed via HPLC [27]. The anthocyanin content was measured as described previously [28].

For the measurement of total free amino acids, the ninhydrin colorimetric method was used. Briefly, a $3 \mathrm{~g}$ dried sample was extracted in a boiling water bath for $45 \mathrm{~min}$ in $450 \mathrm{ml}$ of $\mathrm{H}_{2} \mathrm{O}$ (with shaking once every $10 \mathrm{~min}$ ). After filtration, the volume of the filtrates was increased to $500 \mathrm{ml}$ by adding $\mathrm{H}_{2} \mathrm{O}$, and $1 \mathrm{ml}$ of the solution was transferred to a $25 \mathrm{ml}$ flask. Following the addition of $0.5 \mathrm{ml}$ of buffer ( $\mathrm{pH} 8.0$ ) containing $63 \mathrm{mM}$ $\mathrm{Na}_{2} \mathrm{HPO}_{4}$ and $3 \mathrm{mM} \mathrm{KH} \mathrm{PO}_{4}, 0.5 \mathrm{ml}$ of a $2 \%$ ninhydrin solution ( $2 \mathrm{~g}$ ninhydrin and $80 \mathrm{mg} \mathrm{SnCl}_{2} \cdot 2 \mathrm{H}_{2} \mathrm{O}$ dissolved in $100 \mathrm{ml}$ of water) was added, and the flask was incubated in a boiling water bath for $15 \mathrm{~min}$. The volume was then increased to $25 \mathrm{ml}$ with $\mathrm{H}_{2} \mathrm{O}$. The absorption values of the solution at $570 \mathrm{~nm}$ were determined using a spectrophotometer. The total free amino acid content was calculated from a standard curve generated with varying concentrations of glutamine.

\section{Transmission electron microscopic (TEM) analysis}

The ultrastructure of the chloroplasts was investigated via TEM according to the method described by $\mathrm{Du}$ et al. [5]. Leaf samples (fresh shoots) were cut into small pieces and fixed with $2.5 \%$ glutaraldehyde overnight at $4 \mathrm{C}$. The samples were then washed with $0.1 \mathrm{M}$ phosphate buffer
(pH 7.0) three times (15 min each) and subsequently refixed in $1 \%(\mathrm{v} / \mathrm{v}) \mathrm{OsO}_{4}$ for $2 \mathrm{~h}$ and washed with $0.1 \mathrm{M}$ phosphate buffer again. For dehydration, the fixed samples were subjected to a graded ethanol series $(50 \%, 70 \%, 80 \%$, 90\% and 95\%), with each dehydration step lasting $15 \mathrm{~min}$, and then soaked in $100 \%$ ethanol for $20 \mathrm{~min}$. The dehydrated samples were drenched in acetone for $20 \mathrm{~min}$, followed by epoxy resin and acetone $(\mathrm{v} / \mathrm{v}=3 / 1)$ for $1 \mathrm{~h}$. The samples were finally imbedded in pure epoxy resin at 70C overnight.

After imbedding, $7090 \mathrm{~nm}$ thick sections were cut with a Reichert-Jung ultra-cut microtome (ReichertJung, Heidelberg, Germany). The sections were stained with saturated uranyl acetate in 50\% ethanol and $0.2 \%$ $(\mathrm{w} / \mathrm{v})$ lead citrate for $15 \mathrm{~min}$ each. The images were examined under a JEM-1230 microscope (JEOL, Akishima, Tokyo, Japan).

\section{Microarray data analysis}

Total RNA was extracted from $0.5 \mathrm{~g}$ to $1 \mathrm{~g}$ of leaf samples from $\mathrm{ZH} 2$ and LJ43 tea plants as described previously [29] for microarray and qRT-PCR assays. The 60-mer oligonucleotide probes and microarray were designed by eArray (Agilent). The probes on the microarray were based on the 42,440 unigene sequences obtained from a 454 RNA-seq dataset in our laboratory [24]. The microarray data were deposited into the NCBI Gene Expression Omnibus (GEO) database under accession number GSE52255 [24].

Feature Extraction Software (10.7.1.1, Agilent Technologies) was used to analyze array images to obtain raw data. GeneSpring was employed to complete the basic analysis of the raw data. The raw data were normalized with a quantile algorithm. The probes with at least one condition out of every condition flagged in $P$ were chosen for further data analysis. Differentially expressed genes were subsequently identified based on the observed fold changes and using a $t$-test-calculated $P$-value. A differentially expressed gene was defined as a variation in the gene expression test showing a $P$-value $<0.05$ and a fold change $>2$. Significant pathways were identified based on the Kyoto Encyclopedia of Genes and Genomes (KEGG). Fisher s exact test was employed to select pathways, and the threshold of significance was defined as a $P$-value $<0.05$ and an false discovery rate $(\mathrm{FDR})<0.05$.

\section{Quantitative Real-Time RT-PCR}

Five micrograms of RNA was used to synthesize cDNA. The RNA samples were treated with RNase-free DNase I (amplification grade, Invitrogen, Carlsbad, CA, USA) to remove residual genomic DNA. First-strand cDNA was synthesized using SuperScript III Reverse Transcriptase (Invitrogen, Carlsbad, CA, USA). Real-time quantitative RT-PCR was performed with the Applied 
Biosystems 7500 Sequence Detection System (Carlsbad, CA, USA) using SYBR Premix Ex Taq II (TaKaRa Biomedicals). The amplification efficiency of the primers for the tested genes is shown in Additional file 1. Triplicate quantitative assays were performed on each cDNA sample, and the $18 \mathrm{~S}$ rRNA reference gene was used as an internal control. Relative transcript levels were calculated relative to the level of $18 \mathrm{~S}$ rRNA using the $2^{-\Delta \Delta C t}$ formula [30]. All data are presented as the mean SD $(n=3)$. All of the primer sequences used for qRT-PCR are shown in Additional file 2.

\section{Statistical analysis of the data}

The data were expressed as the means SD from three independent biological replicates. Significance was determined via one-way analysis of variance, and for differences between groups, the least significant difference (LSD) $t$ test was employed $(P<0.05)$.

\section{Results}

Growth performance of a chlorophyll-deficient chlorina cultivar

A yellow-leaf cultivar was developed from a natural mutant and was designated $\mathrm{ZH} 2$. In spring, $\mathrm{ZH} 2$ exhibits yellow new shoots, which differs from what occurs in LJ43, a green tea cultivar that is widely planted in China (Figure 1A and B). It is well known that leaf greening is the result of chlorophyll biosynthesis, and the chlorophyll contents of two leaves and one bud of the tea plants were measured. The results showed that in $\mathrm{ZH} 2$, both chlorophyll $a$ and chlorophyll $b$ contents were significantly lower than in LJ43 (approximately 14\% and
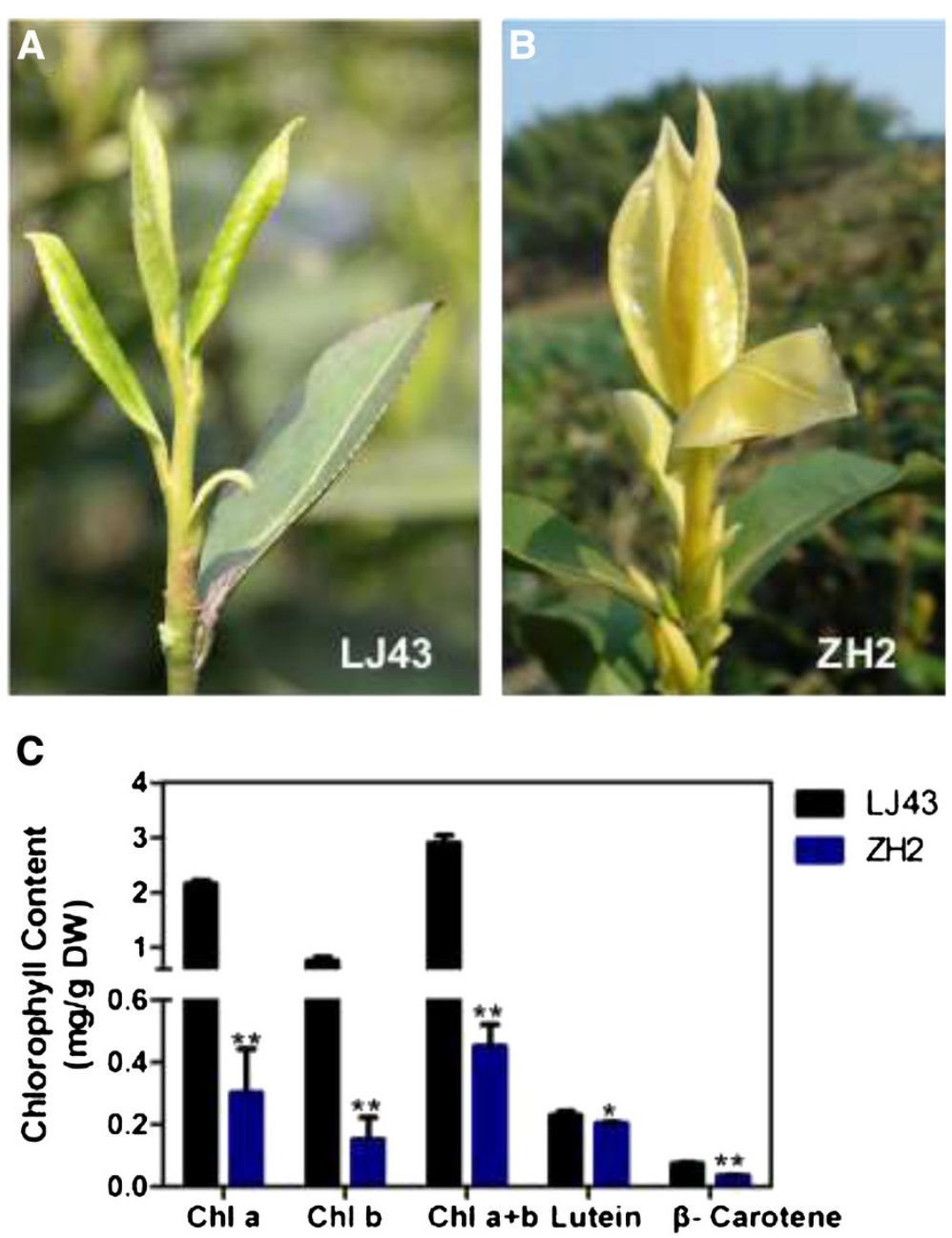

Figure 1 Growth performance and pigment (chlorophyll and carotenoid) contents of $\mathrm{L} \mathbf{4 3}$ and ZH2. (A) Growth performance of $L 443$ shoots in spring. (B) Growth performance of ZH2 shoots in spring. (C) Chlorophyll $a$ (Chl a), chlorophyll $b$ (Chl b), Chl $a+b$, lutein and $\beta$-carotene contents of two leaves and one bud of $\mathrm{L} 43$ and Z $\mathrm{H} 2$. The significance of differences compared with $\sqcup 43$ is indicated with an asterisk $(P<0.05)$ or two asterisks $(P<0.01)$. 
$20 \%$ of the contents in LJ43, respectively; Figure 1C). These results suggested that the yellow leaves of $\mathrm{ZH} 2$ result from reduced chlorophyll levels and that the lower chlorophyll content of $\mathrm{ZH} 2$ might result from abnormal chlorophyll biosynthesis.

Carotenoids are lipid-soluble pigments that are essential components of the photosynthetic apparatus. Beta-carotene and lutein, the major carotenoids found in tea leaves, are two nutritionally important plantderived carotenoids [31]. The rice pigment-deficient mutant $y l c 1$ exhibits a chlorosis phenotype with decreased levels of chlorophyll and lutein [22]. Betacarotene and lutein contents were measured in the two cultivars. The results showed that in the young shoots of $\mathrm{ZH} 2$, the beta-carotene and lutein contents were decreased to $48.8 \%$ and $88.6 \%$ of the levels in LJ43, respectively (Figure $1 \mathrm{C}$ ).

\section{Ultrastructure of chloroplasts in LJ43 and $\mathrm{ZH} 2$}

Photosystems involve chlorophyll and other pigments, which are located in the grana. The saclike membranes that make up grana are known as thylakoids. To understand why yellow leaves occur in $\mathrm{ZH} 2$, the ultrastructure of the chloroplast, which is responsible for leaf greening, was investigated through TEM. TEM analysis showed a typical ultrastructure, consisting of grana and thylakoids, in the chloroplasts of LJ43 plants (Figure 2A to C). However, in the chloroplasts of $\mathrm{ZH} 2$, the stacks of grana disappeared, and only a few thylakoids remained (Figure 2D to $F$ ). This result indicated that the change in the leaf color of $\mathrm{ZH} 2$ might be a consequence of damage to the development of grana.

\section{Theanine, amino acid and flavonoid contents of LJ43 and $\mathrm{ZH} 2$}

Amino acids are important in the sensory quality of green tea [32]. Theanine, which was first discovered in tea leaves, is the most abundant free amino acid in tea plants and is beneficial for human health [33-37]. The theanine and total free amino acids contents in the shoots (two leaves and one bud) of LJ43 and $\mathrm{ZH} 2$ were analyzed. The results showed that the contents of both theanine and total free amino acids were significantly higher in the leaves of $\mathrm{ZH} 2$ than in LJ43 (Figure 3A).

Flavonoids are important for tea quality and are synthesized through a branched pathway that yields both colorless compounds (e.g., flavonols) and colored pigments (e.g., anthocyanins) [38]. Catechins represent $60 \%$ to $80 \%$ of the total flavonoids in green tea [39]. Measurement of the contents of catechins and anthocyanins in

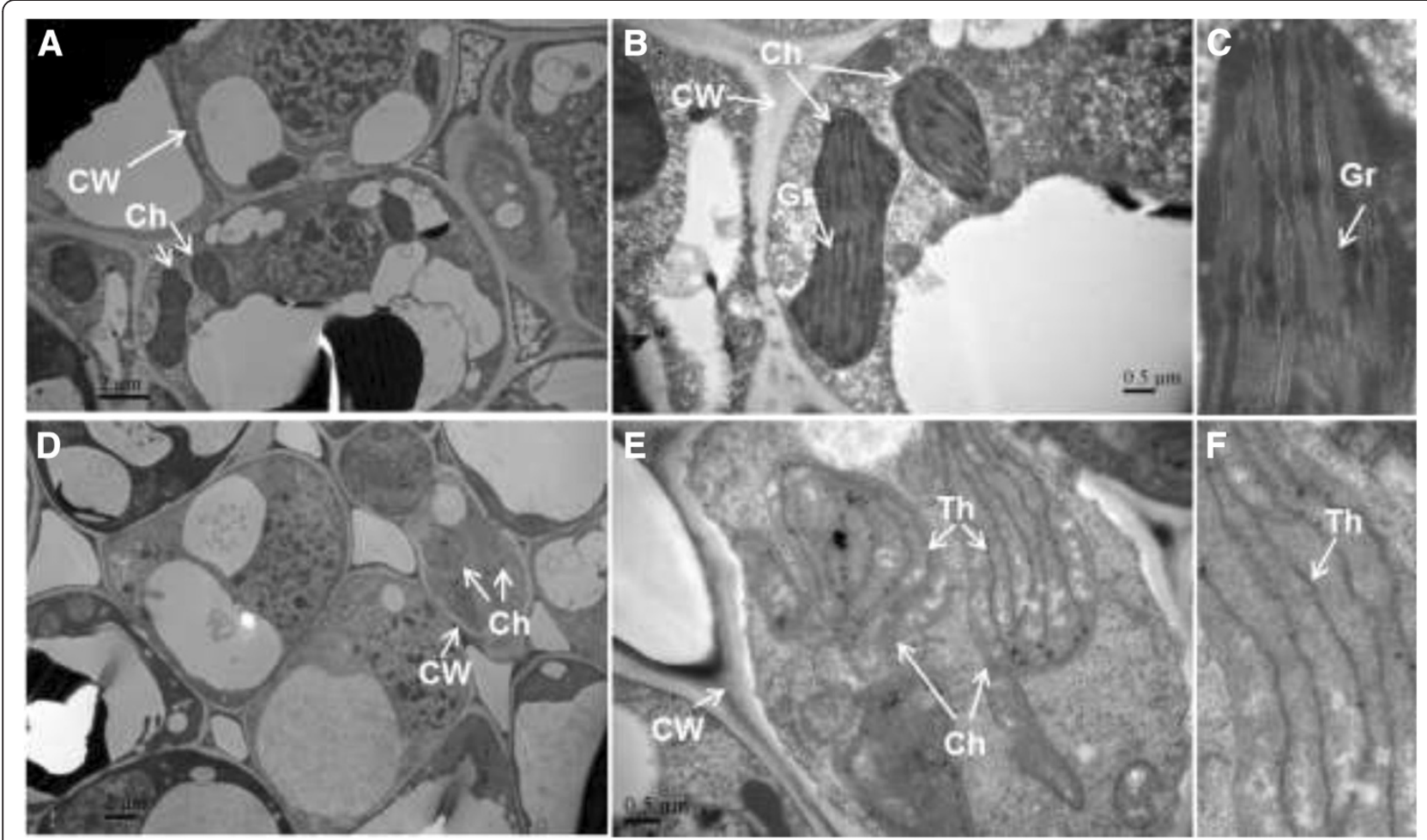

Figure 2 Comparison of subcellular structures in the young leaves of LJ43 and ZH2. (A, B, C) Subcellular structures of LJ43. (D, E, F) Subcellular structures of ZH2. (C, F) Magnification of the chloroplasts from B and E, respectively. Ch: Chloroplast, CW: Cell Wall, Gr: Grana, Th: Thylakoid. 

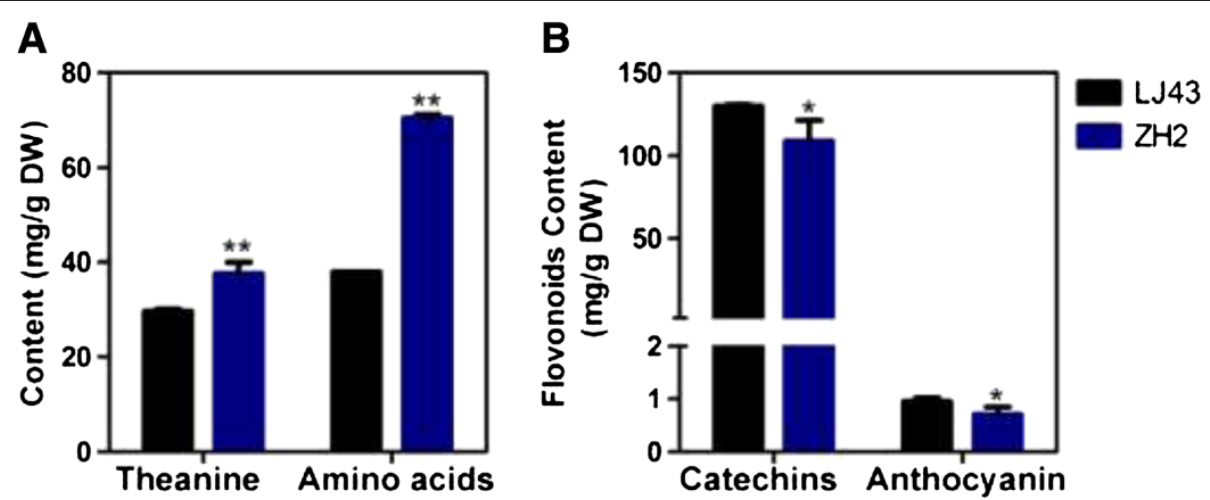

Figure 3 Theanine, amino acid, catechins and anthocyanin contents of LJ43 and ZH2. (A) Theanine and amino acid contents of two leaves and one bud of the two cultivars. (B) Catechins and anthocyanin contents in two leaves and one bud of the two cultivars. The significance of differences compared with LJ43 is indicated with an asterisk $(P<0.05)$ or two asterisks $(P<0.01)$.

shoots revealed that they were lower in ZH2 than in LJ43 (Figure 3B).

\section{Microarray analysis of gene expression}

To investigate the performance of $\mathrm{ZH} 2$, the differences in gene expression between $\mathrm{ZH} 2$ and LJ43 were studied to elucidate the reason for the different phenotype of $\mathrm{ZH} 2$ plants. A custom microarray was used to assess global gene expression in $\mathrm{ZH} 2$ and LJ43. The number of genes that were significantly $(P<0.05)$ differentially expressed in $\mathrm{ZH} 2$ by more than 2 fold compared with LJ43 are listed in Figure 4A. Altogether, 4,902 differentially expressed genes were identified, including 2,308 genes that were up-regulated and 2,594 that were downregulated in $\mathrm{ZH} 2$ (Figure 4A). Genes related to amino acid metabolism, pigment (chlorophyll, carotenoid and flavonoid) metabolism and photosynthesis are indicated in the blue circle (125 up-regulated and 134 downregulated) (Figure 4A, Additional file 3).

An evaluation of functional category enrichment based on gene ontology (GO) was performed on the genes that were differentially expressed $(P$-value $<0.05$, fold-change $>2$ ) between ZH2 and LJ43. Significant GO terms were determined at a cut-off $P$-value $<0.05$. AgriGO annotated 3,182 out of 4,902 differentially expressed genes with at least one significant GO term, and the annotated probes were classified according to the biological process, molecular function and cellular component categories and their sub-categories (Table 1). Among the three components, the metabolic process sub-category of the biological process category accounted for the majority of GO annotations, followed by binding under molecular function (Table 1).

To identify biological pathways, the genes were annotated with the corresponding enzyme commission (EC) numbers from BLASTX alignments against the KEGG database. By associating the 4,902 differentially expressed genes with Gene IDs in KEGG, 255 pathways were mapped (Additional file 4). Among these pathways, 24 pathways, including cysteine and methionine metabolism, glycine, serine and threonine metabolism, flavonoid biosynthesis, porphyrin and chlorophyll metabolism and carotenoid biosynthesis, were considered significant at a cut-off $P$-value $<0.05$ and FDR $<0.05$ (Figure $4 \mathrm{~B}$ ). These pathways are suggested to be important to the unusual phenotype of $\mathrm{ZH} 2$. The 259 genes (Additional file 3) from the up- and down-regulated datasets presented in Figure 4A (blue circle) were classified into 12 pathways, and the enrichment of each pathway is shown in Figure 4C.

When pathways of interest were examined, several differentially expressed genes could be mapped to the theanine biosynthesis, chlorophyll biosynthesis and flavonoid biosynthesis pathways. Theanine is synthesized from glutamic acid and ethylamine by theanine synthetase $(T S)$ [40]. Ethylamine appears to be produced from alanine by alanine decarboxylase (AIDA) [40]. TS and AIDA were not found in our dataset. The AIDA shown in Figure 5A was selected from the homologues of arginine decarboxylase $(A D C)$, which exhibits similar domains to AIDA [41,42]. Alanine transaminase $(A L T)$ and AIDA were significantly $(P$-value $<0.05)$ up-regulated in $\mathrm{ZH} 2$, indicating the possibility that the higher contents of theanine found in $\mathrm{ZH} 2$ resulted from higher transcription levels of the two genes involved in theanine biosynthesis (Figure 5A).

Nine genes in the chlorophyll biosynthesis pathway showed differential expression $(P$-value $<0.05$, fold change $>2$; 4 up-regulated and 5 down-regulated genes). The expression of some critical successive enzymes for converting 5-amino-levulinate (ALA) to chlorophyll $a$ was altered to variable extents in $\mathrm{ZH} 2$ (Figure $5 \mathrm{~B}$ ). $\mathrm{ChlH}$, which is a subunit of Mg-chelatase, was up-regulated in ZH2. Transcripts encoding enzymes such as those functioning in early enzymatic steps, from the formation of glutamate- 


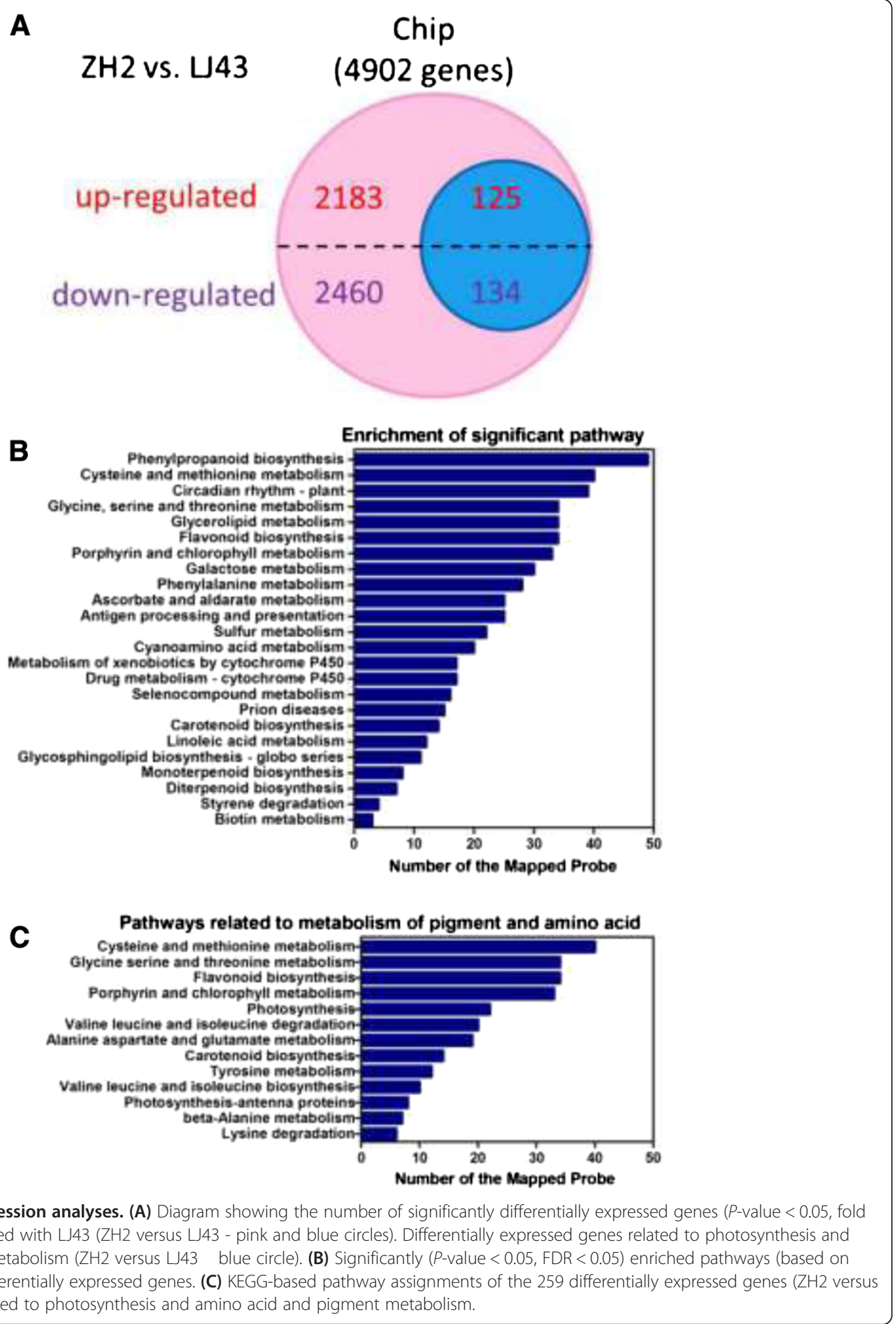

1-semialdehyde to protoporphyrin IX, showed lower levels. Critical enzymes for converting Mg-protoporphyrin IX to chlorophyll were also inhibited (Figure 5B).
Compared with LJ43, ZH2 exhibited lower contents of catechins and anthocyanin (Figure 3B). To examine whether the expression of genes related to flavonoid 
Table 1 Categorization of differentially expressed genes between ZH2 and LJ43

\begin{tabular}{|c|c|c|c|c|}
\hline \multirow[t]{2}{*}{ GO terms } & \multicolumn{3}{|c|}{ Differentially expressed genes } & \multirow{2}{*}{$\begin{array}{l}\text { Ratio } \\
\text { (\%) }\end{array}$} \\
\hline & Total & Up-regulated & Down-regulated & \\
\hline \multicolumn{5}{|l|}{ Cellular component } \\
\hline GO: 0016020 membrane & 924 & 436 & 488 & 29.04 \\
\hline GO: 0005623 cell & 485 & 226 & 259 & 15.24 \\
\hline GO: 0005576 extracellular region & 130 & 41 & 89 & 4.09 \\
\hline GO: 0030054 cell junction & 1 & 1 & 0 & 0.03 \\
\hline \multicolumn{5}{|l|}{ Biological process } \\
\hline GO: 0008152 metabolic process & 1758 & 820 & 938 & 55.25 \\
\hline GO: 0051179 localization & 493 & 232 & 261 & 15.49 \\
\hline GO: 0050896 response to stimulus & 289 & 113 & 176 & 9.08 \\
\hline GO: 0009987 cellular process & 331 & 146 & 185 & 10.4 \\
\hline GO: 00650007 biological regulation & 196 & 98 & 98 & 6.16 \\
\hline GO:0071840 cellular component organization or biogenesis & 33 & 9 & 24 & 1.04 \\
\hline GO:0032502 developmental process & 20 & 9 & 11 & 0.63 \\
\hline GO:0051704 multi-organism process & 5 & 3 & 2 & 0.16 \\
\hline GO:0032501 multicellular organismal process & 4 & 0 & 4 & 0.13 \\
\hline \multicolumn{5}{|l|}{ Molecular function } \\
\hline GO: 0005488 binding & 1010 & 473 & 537 & 31.74 \\
\hline GO: 0005215 transporter activity & 116 & 62 & 54 & 3.65 \\
\hline GO: 0009055 electron carrier activity & 112 & 44 & 68 & 3.52 \\
\hline GO: 0016209 antioxidant activity & 26 & 16 & 10 & 0.82 \\
\hline GO: 0045735 nutrient reservoir activity & 18 & 11 & 7 & 0.57 \\
\hline GO: 0005198 structural molecule activity & 1 & 0 & 1 & 0.03 \\
\hline
\end{tabular}

Note that an individual probe might be assigned to more than one GO term.

biosynthesis was altered in $\mathrm{ZH} 2$, we searched the differentially expressed genes using EC numbers and found that genes involved in flavonoid biosynthesis were significantly down-regulated in $\mathrm{ZH} 2$ (Figure $5 \mathrm{C}$ ), which is consistent with the lower catechins and anthocyanin contents observed in the young shoots of $\mathrm{ZH} 2$.

\section{Quantitative RT-PCR validation of some differentially expressed genes}

To confirm the microarray data and the expression changes in genes related to chlorophyll biosynthesis, theanine biosynthesis and flavonoid biosynthesis, qRTPCR was performed. The expression of 23 genes (including 2 genes involved in theanine biosynthesis, 9 genes involved in chlorophyll biosynthesis and 12 genes involved in flavonoid biosynthesis) was detected. The expression of 19 genes detected via qRT-PCR showed a pattern that was similar to that observed in the microarray data, whereas 4 genes (CsNOL, CsPAL, CsFNS, CsF3H) showed a different expression pattern (Figure 6). Ten genes randomly selected from these 23 genes were cloned by RACE to support that the differentically expressed genes detected by qRT-PCR in the two cultivars were believable (Additional file 5). Overall, the qRT-PCR results were well correlated with the microarray data.

\section{Discussion}

Tea cultivars exhibiting unique color changes have drawn increasing attention due to the differences in the chemical composition of their leaves. In this study, we report a novel chlorophyll-deficient chlorina tea cultivar that presents a yellow new shoot phenotype (Figure 1). The ZH2 cultivar was selected during tea plant breeding, and genetic and biochemical characterization of $\mathrm{ZH} 2$ was performed.

Pigment analysis showed that the young shoot phenotype of $\mathrm{ZH} 2$ is positively correlated with the levels of chlorophyll, beta-carotene and lutein (Figure 1). It seemed that the arrested development of grana in the chloroplasts blocks chlorophyll and carotenoids biosynthesis in $\mathrm{ZH} 2$ and the photosynthesis was inhibited in $\mathrm{ZH} 2$ leaves. Recently, Feng et al. found that decreased abundances of carotenoids and chlorophylls were accompanied by an increase in the abundances of free amino acids in albino tea 


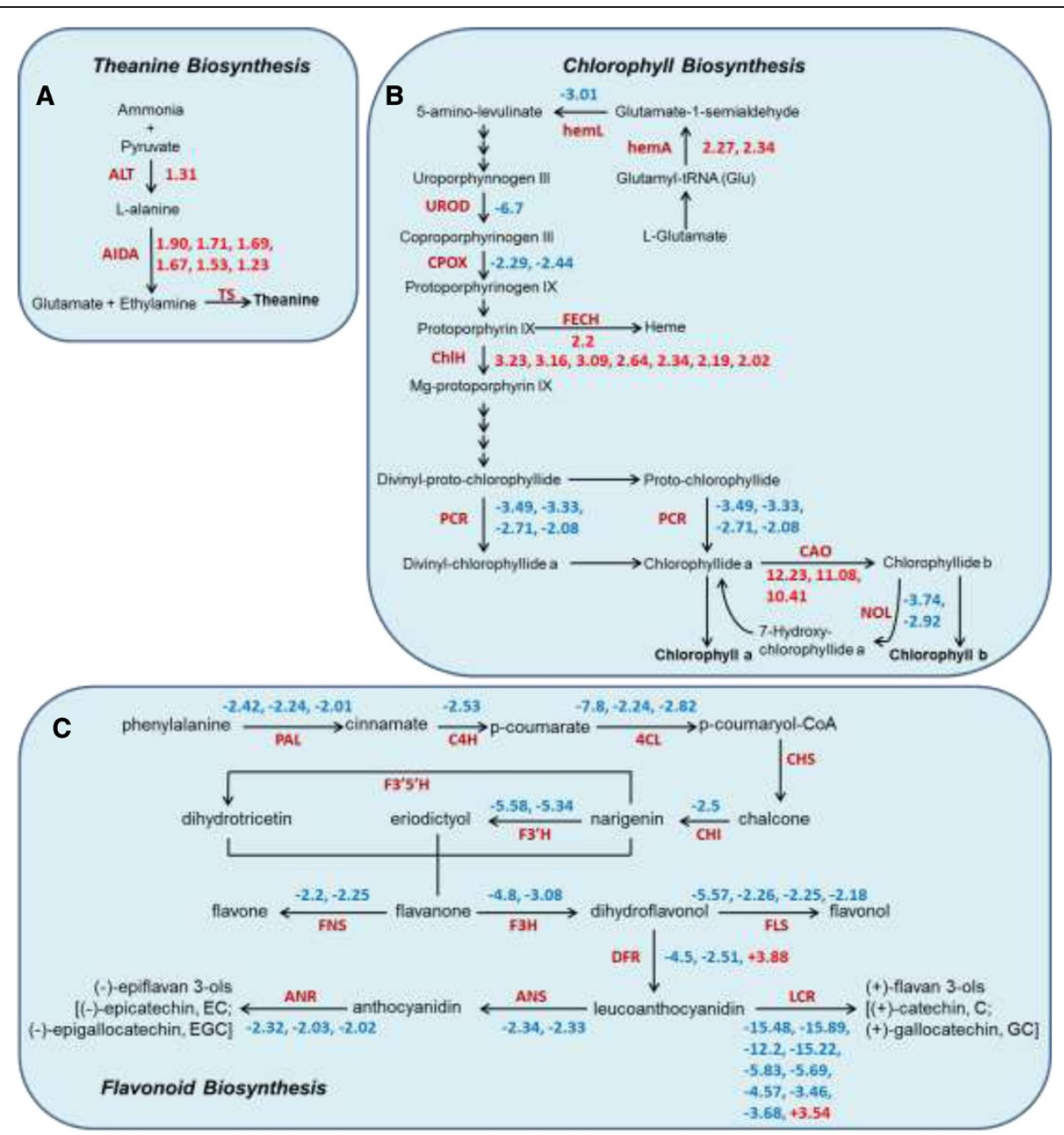

Figure 5 Schematic representation of significant changes in the transcript levels of genes involved in three pathways. (A) Genes involved in the theanine biosynthesis pathway (note that AIDA was selected from the homologues of arginine decarboxylase, ADC). (B) Genes involved in the chlorophyll biosynthesis pathway. (C) Genes involved in the flavonoid biosynthesis pathway. The flavonoid biosynthesis pathway was drawn according to Shi et al. [41]. The numbers represent the fold change; positive numbers indicate significantly $(P<0.05)$ up-regulated genes, while negative numbers indicate significantly $(P<0.05)$ down-regulated genes.

leaves [43]. This finding is in accordance with our results, which showed that the contents of chlorophyll and the carotenoids of beta-carotene and lutein were decreased in ZH2 (Figure 1C). Chlorophylls and carotenoids are important for the biogenesis of the photosynthetic apparatus. Carotenoids are synthesized in the membranes of nearly all types of plastids, including chloroplasts $[44,45]$. In chloroplasts, carotenoids form photosynthetic complexes in thylakoid membranes, and they play an important role in protecting the chlorophyll from destruction $[46,47]$. Thus, it is reasonable that reduction in levels of carotenoids were accompanied with the reduction in levels of chlorophylls and the abnormal pigments contents were along with the aberrant development of chloroplast. Also, the result will be helpful for furthering our understanding the chlorina phenomenon in relation to carotenoid biosynthesis and the development of the photosynthetic apparatus.

Changes in chlorophyll contents may be one reason for yellow leaves. Changes in the expression of genes involved in chlorophyll biosynthesis might result in the chlorina phenotype of $\mathrm{ZH} 2$. Mg-chelatase plays an important role in chlorophyll production. In plants, this enzyme complex consists of three subunits, designated ChlD, ChlH and ChlI. Mg-chelatase deficiency is a common denominator in many chlorophyll-deficient mutants. Plants lacking $\mathrm{ChlH}$ exhibit defective chlorophyll and show a chlorina phenotype $[9,10,20]$. Although $\mathrm{ZH} 2$ presents a leaf phenotype that is similar to mutants such as Oschlh, the expression of $\mathrm{CsChlH}$ was found to be up-regulated in $\mathrm{ZH} 2$ (Figures 5B and 6A). Considering the other two subunits of Mg-chelatase, ChlD and ChlI, 


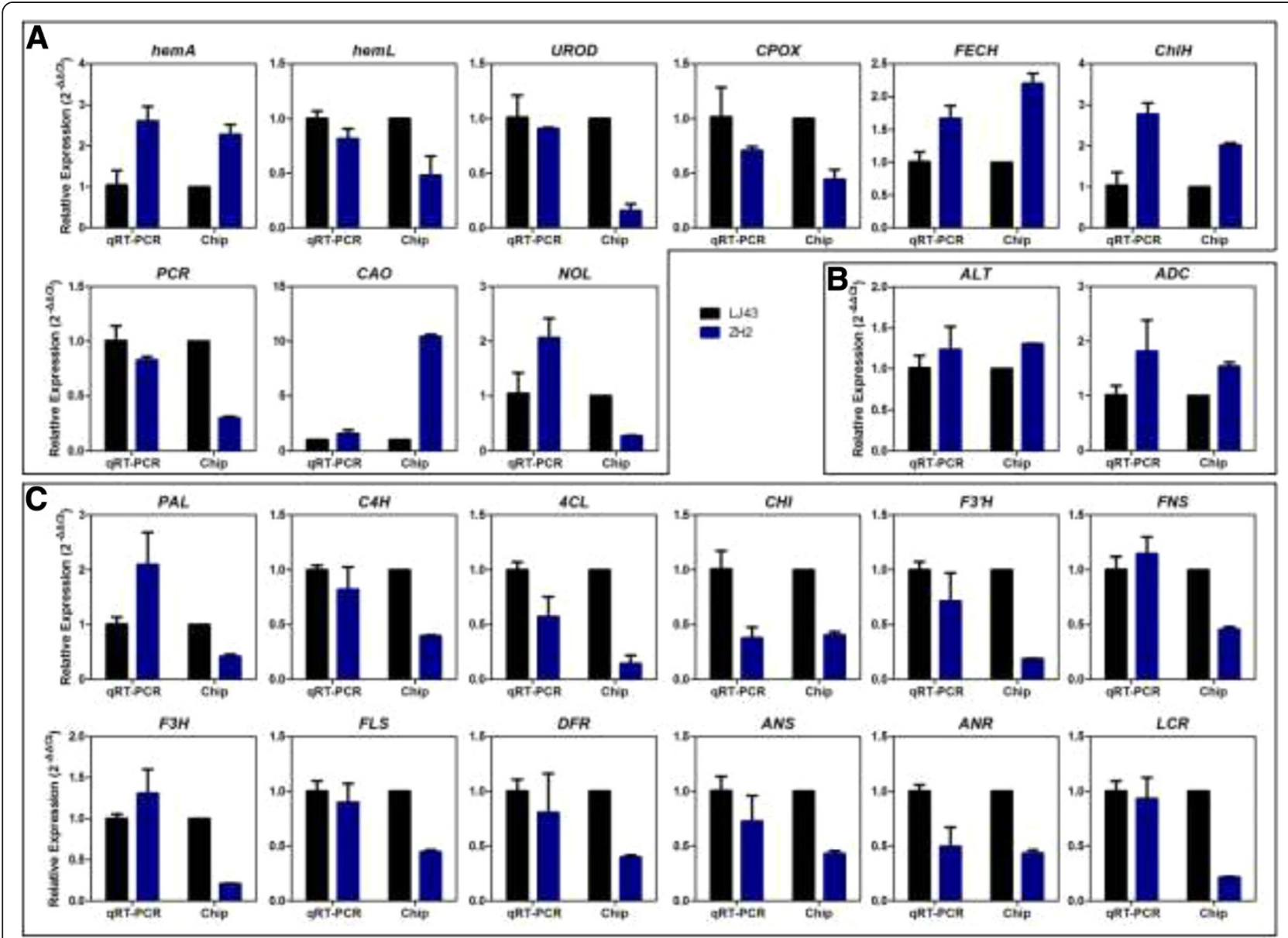

Figure 6 Gene expression patterns in the shoots of LJ43 and ZH2. (A) Genes involved in the chlorophyll biosynthesis pathway. (B) Genes involved in the theanine biosynthesis pathway. (C) Genes involved in the flavonoid biosynthesis pathway. All data are shown as the mean SD $(n=3)$.

which, when mutated, can also cause a chlorina phenotype in plants [11,19], we examined the expression of CsChlD and CsChlI in the microarray data. We found that CsChlI (ZH2 versus LJ43, P-value $=0.011$, fold change $=1.16)$ and CsChlD (ZH2 versus LJ43, $P$-value $=0.000031$, fold change $=1.27)$ were also slightly up-regulated in $\mathrm{ZH} 2$. This result indicated that the expression of the three subunits of $\mathrm{Mg}$-chelatase was not disrupted in $\mathrm{ZH} 2$ and that the chlorina phenotype of $\mathrm{ZH} 2$ was not correlated with a lack of $\mathrm{Mg}$-chelatase subunits, as has been reported in other plants. However, it is likely that there were changes in these genes at the posttranscriptional level that might affect the $\mathrm{ZH} 2$ phenotype.

In Camellia sinensis, Ma (2012) analyzed differentially expressed genes in different albescent stages of the Anji Baicha cultivar using a $5 \mathrm{~K}$ cDNA microarray and found that $\mathrm{Cs} C h l \mathrm{H}$ was up-regulated at the pale white shoot stage, consistent with our findings [48]. It has been reported that both decreased and increased expression of ChlI decreases Mg-chelatase activity and reduces chlorophyll synthesis in transgenic tobacco plants [21]. Active Mg-chelatase requires a balanced proportion of each subunit, and an excess of the ChlI subunit could disturb the correct assembly of the enzyme complex [21]. In the present study, $\mathrm{Cs} C h l \mathrm{H}$ showed a higher level of induction than CsChlD and CsChlI in $\mathrm{ZH} 2$, which might upset the balance of the three subunits and consequently influence $\mathrm{Mg}$-chelatase activity in $\mathrm{ZH} 2$.

Genes encoding enzymes involved in the early enzymatic steps of the chlorophyll biosynthesis pathway were found to be suppressed in ZH2 (Figure 5B). Lower activities related to ALA synthesis and the resulting lower protoporphyrin IX contents are directly correlated with reduced $\mathrm{Mg}$-chelatase activity [49]. CAO is required for chlorophyll $b$ synthesis, and the expression of $C A O$ is up-regulated in plants that do not have sufficient chlorophyll $b$ [50]. The present study revealed an increased transcript level of the 
CsCAO gene in $\mathrm{ZH} 2$, which is consistent with its lower chlorophyll $b$ content (Figures $1 \mathrm{C}$ and 5B). Interestingly, the chlorina phenotype of $\mathrm{ZH} 2$ disappeared under shade treatment (unpublished data) and we speculate that the chlorina phenotype with chlorophyll metabolic disorder in ZH2 may be regulated by light. Shade treatment could be performed to investigate the effect of low light on the chlorina phenotype and gene expression pattern in the future.

Based on microarray analysis, differentially expressed genes were identified. Twenty-four pathways were identified as significantly regulated, many of which were related to amino acid metabolism and pigment metabolism, which was strongly correlated with the different chlorophyll and amino acid contents observed in ZH2 (Figure 4B). Furthermore, many secondary metabolic processes, such as flavonoid biosynthesis, that are important for plant growth and development were significantly enriched [51]. It has been reported that the catechins content decreases during the albescent process in tea plants, which is consistent with our findings [43,52]. Genes involved in flavonoid biosynthesis were repressed in $\mathrm{ZH} 2$ (Figure $5 \mathrm{C}$ ). This repression might be responsible for the lower amounts of the anthocyanin pigments and catechins found in ZH2 (Figure 3B). Anthocyanin/flavonoids are responsible for the red, blue and purple anthocyanin pigments of plant tissues [53]. It is possible that the decreased levels of anthocyanin pigments might be related to the changes in leaf color observed in $\mathrm{ZH} 2$. Several secondary metabolic processes, though not flavonoid biosynthesis, were also significantly enriched (Figure 4B), which suggested that the secondary metabolites of $\mathrm{ZH} 2$ were altered, and it is therefore of interest to study the metabolic profile of $\mathrm{ZH} 2$ in the future.

In this study, we compared biochemical components and the transcriptomes of one chlorina cultivar ( $\mathrm{ZH} 2)$ and one normal green tea plant cultivar (LJ43) and found that there were many differences in biochemical components as well as in transcriptome profiles. Microarray analysis revealed that some differentially expressed genes could be mapped to the theanine biosynthesis, chlorophyll biosynthesis and flavonoid biosynthesis pathways based on KEGG. Changes in the expression of genes involved in these three pathways might be responsible for the higher theanine content and the chlorina phenotype of $\mathrm{ZH} 2$. Thus, this study provides further insights into the molecular mechanisms underlying the phenotype of this chlorina cultivar of Camellia sinensis. However, it is possible that the genes annotated in this study might not function as expected because the genome sequence information for the tea plant is not available, and we cannot rule out the possibility that our analysis of differentially expressed genes might be influenced by inappropriate annotation. As ZH2 and LJ43 do not share the same genetic background, many of the changes that we identified in this study might be presumptive and preliminary. Nevertheless because phenotypic traits such as the contents of free amino acids, catechins, flavonoids and pigments are controlled by genetic factors, the differences between $\mathrm{ZH} 2$ and LJ43 might result from changes in gene expression. We also found that the chlorina phenotype was heritable in the $F_{1}$ offspring of $\mathrm{ZH} 2$ plants, and the chlorina phenomenon observed in $\mathrm{ZH} 2$ is influenced by light intensity and time. Therefore, the results obtained in this study may provide new insights toward elucidating the essential mechanism underlying the chlorina phenotype of tea plants. In the future, we will carry out further research to elucidate the regulatory factors (e.g., environmental and genetic factors) that control and regulate the chlorina phenotype of $\mathrm{ZH} 2$.

\section{Conclusions}

In summary, the physiological characteristics of the novel chlorophyll-deficient chlorina cultivar Zhonghuang 2 (ZH2) were analyzed, and gene expression profiling was performed using a 4X 44 K custom oligonucleotide-based microarray (GSE52255). The lower chlorophyll contents and abnormal ultrastructure of chloroplasts observed in the leaves of ZH2 suggested that chlorophyll biosynthesis was partially inhibited. As observed in other leaf color mutant cultivars of Camellia sinensis, $\mathrm{ZH} 2$ exhibited higher contents of theanine and free amino acids. Differential expression analysis revealed 4,902 genes that were differentially expressed in the two cultivars, and 24 pathways were identified as significantly differentially regulated, including amino acid metabolism and pigment metabolism pathways, which might be related to the higher amino acid content and chlorophyll-deficient phenotype of $\mathrm{ZH} 2$. Further analysis revealed that a number of differentially expressed genes could be mapped to the theanine biosynthesis, chlorophyll biosynthesis and flavonoid biosynthesis pathways based on KEGG. Changes in the expression of genes involved in these three pathways might be responsible for the higher theanine content and the chlorina phenotype of $\mathrm{ZH} 2$. Our study provides further insights into the molecular mechanisms underlying the phenotype of this chlorina cultivar of Camellia sinensis.

\section{Additional files}

Additional file 1: Amplification efficiency of the genes tested by qRT-PCR.

Additional file 2: Primer sequences for quantitative RT-PCR.

Additional file 3: The $\mathbf{2 5 9}$ differentially expressed genes related to amino acid metabolism, pigment (chlorophyll, carotenoid and flavonoid) metabolism and photosynthesis.

Additional file 4: The 255 pathways that mapped to the differentially expressed genes between $\mathrm{ZH} 2$ and $\mathrm{L} 43$.

Additional file 5: Ten genes randomly selected from microarray data were cloned from two tea cultivars by RACE. 


\section{Abbreviations}

ADC: Arginine decarboxylase; AIDA: Alanine decarboxylase; ALA: Ainolevulinic acid; ALT: Alanine transaminase; ANR: Anthocyanidin reductase; ANS: Anthocyanidin synthase; CAO: Chlorophyllide a oxygenase; C4H: Cinnamate 4-hydroxylase; $\mathrm{CHI}$ : Chalcone isomerase; ChlH: Magnesium chelatase subunit H; CHS: Chalcone synthase; 4CL: 4-coumarate CoA ligase; CPOX: Coproporphyrinogen oxidase; DFR: Dihydroflavonol 4-reductase; EC: Enzyme commission; FDR: False discovery rate; FECH: Ferrochelatase; F3H: Flavanone 3-hydroxylase; F3'H: Flavonoid 3'-hydroxylase; F3'5'H: Flavonoid 3',5'-hydroxylase; FLS: Flavonol synthase; FNS: Flavone synthase; GEO: Gene expression omnibus; GO: Gene ontology; hemA: Glutamyl-tRNA reductase; hemL: Glutamate-1-semialdehyde aminotransferase; KEGG: Kyoto encyclopedia of genes and genomes; LCR: Leucoanthocyanidin reductase; NOL: Chlorophyll b reductase; PAL: Phenylalanine ammonia lyse; PCR: Protochlorophyllide reductase; TEM: Transmission electron microscopic; TS: Theanine synthetase; UROD: Uroporphyrinogen III decarboxylase.

\section{Competing interests}

The authors declare that they have no competing interests.

\section{Authors contributions}

XCW and YJY participated in the design and coordination of the study. LW, CY, $\mathrm{HLC}, Y H Z$ and $J M Z$ did the biochemical and gene expression analysis. LW, CY and XCW wrote the paper. All authors read and approved the final manuscript.

\section{Acknowledgements}

This work was supported by the Earmarked Fund for China Agriculture Research System (CARS-23), the Major Project for New Agricultural Varieties Breeding of Zhejiang Province (2012C2905-3), and the Project for Issues Concerning Agriculture, Countryside and Farmers of Zhejiang Province (2013 11). The authors thank Ms Jing Feng in Shanghai OE Biotech Co. Ltd for assistance with analyzing the data.

\section{Author details}

${ }^{1} T$ Tea Research Institute, Chinese Academy of Agricultural Sciences, Hangzhou 310008, China. ${ }^{2}$ National Center for Tea Plant Improvement, Hangzhou 310008, China. ${ }^{3}$ Key Laboratory of Tea Biology and Resources Utilization, Ministry of Agriculture, Hangzhou 310008, China.

Received: 23 June 2014 Accepted: 25 November 2014 Published online: 10 December 2014

\section{References}

1. Chen L, Zhou ZX, Yang YJ: Genetic improvement and breeding of tea plant (Camellia sinensis) in China: from individual selection to hybridization and molecular breeding. Euphytica 2007, 154(1 2):239 248.

2. Chen J, Wang P, Xia Y, Xu M, Pei S: Genetic diversity and differentiation of Camellia sinensis $\mathrm{L}$. (cultivated tea) and its wild relatives in Yunnan province of China, revealed by morphology, biochemistry and allozyme studies. Genet Resour Crop Evol 2005, 52(1):41 52.

3. Wachira F, Tanaka J, Takeda Y: Genetic variation and differentiation in tea (Camellia sinensis) germplasm revealed by RAPD and AFLP variation. J Horticultural Science and Biotechnology 2001, 76:557 563.

4. Du Y, Liang Y, Wang H, Wang K, Lu J, Zhang G, Lin W, Li M, Fang Q: A study on the chemical composition of albino tea cultivars. J Horticultural Science and Biotechnology 2006, 81(5):809 812.

5. Du Y, Chen H, Zhong W, Wu L, Ye J, Lin C, Zheng X, Lu J, Liang Y: Effect of temperature on accumulation of chlorophylls and leaf ultrastructure of low temperature induced albino tea plant. Afr J Biotechnol 2008, 7(12):1881 1885

6. Cheng H, Li S, Chen M, Yu F, Yan J, Liu Y, Chen L: Physiological and biochemical essence of the extraordinary characters of Anji Baicha. J Tea Sci 1999, 19:87 92.

7. Li Q, Huang J, Liu S, Li J, Yang X, Liu Y, Liu Z: Proteomic analysis of young leaves at three developmental stages in an albino tea cultivar. Proteome Sci 2011, 9(44):1 12

8. Li S, Cheng H, Fu F, Yan J: The change of amino acid in the stage albinism of White leaf NO. 1.J Tea Sci 1996, 16:153 154.

9. Jung K, Hur J, Ryu C, Choi Y, Chung Y, Miyao A, Hirochika H, An G: Characterization of a rice chlorophyll-deficient mutant using the T-DNA gene-trap system. Plant Cell Physiol 2003, 44(5):463 472.
10. Jensen PE, Petersen BL, Stummann BM, Henningsen KW, Willows RD, Vothknecht UC, Kannangara CG, Wettstein D: Structural genes for Mg-chelatase subunits in barley: Xantha-f, -g and-h. Mol Gen Genet 1996, 250(4):383 394.

11. Zhang H, Li J, Yoo J, Yoo S, Cho S, Koh H, Seo H, Paek N: Rice chlorina-1 and chlorina-9 encode ChID and Chll subunits of Mg-chelatase, a key enzyme for chlorophyll synthesis and chloroplast development. Plant Mol Biol 2006, 62(3):325 337.

12. Wu Z, Zhang X, He B, Diao L, Sheng S, Wang J, Guo X, Su N, Wang L, Jiang L, Wang C, Zhai H, Wan J: A chlorophyll-deficient rice mutant with impaired chlorophyllide esterification in chlorophyll biosynthesis. Plant Physiol 2007, 145(1):29 40.

13. Wettstein D, Gough S, Kannangara C: Chlorophyll biosynthesis. Plant Cell 1995, 7(7):1039 1057.

14. Papenbrock J, Grimm B: Regulatory network of tetrapyrrole biosynthesis studies of intracellular signalling involved in metabolic and developmental control of plastids. Planta 2001, 213(5):667 681.

15. Rudoi A, Shcherbakov R: Analysis of the chlorophyll biosynthetic system in a chlorophyll b-less barley mutant. Photosynth Res 1998, 58(1):71 80.

16. Rodrguez VM, Velasco P, Garrido JL, Revilla P, Ords A, Butrn A: Genetic regulation of cold-induced albinism in the maize inbred line $A 661$. J Exp Bot 2013, 64(12):3657 3667

17. Kannangara CG, Vothknecht UC, Hansson M, von Wettstein D: Magnesium chelatase: association with ribosomes and mutant complementation studies identify barley subunit Xantha-G as a functional counterpart of Rhodobacter subunit BchD. Mol Gen Genet 1997, 254(1):85 92.

18. Papenbrock J, Grfe S, Kruse E, Hnel F, Grimm B: Mg-chelatase of tobacco: identification of a Chl D cDNA sequence encoding a third subunit, analysis of the interaction of the three subunits with the yeast two-hybrid system, and reconstitution of the enzyme activity by co-expression of recombinant CHL D, CHL H and CHL I. Plant J 1997, 12(5):981 990.

19. Rissler HM, Collakova E, DellaPenna D, Whelan J, Pogson BJ: Chlorophyll biosynthesis. expression of a second $\mathrm{Chl}$ I gene of magnesium chelatase in Arabidopsis supports only limited chlorophyll synthesis. Plant Physiol 2002, 128(2):770 779.

20. Mochizuki N, Brusslan J, Larkin R, Nagatani A, Chory J: Arabidopsis genomes uncoupled 5 (GUN5) mutant reveals the involvement of Mg-chelatase $\mathrm{H}$ subunit in plastid-to-nucleus signal transduction. Proc Natl Acad Sci 2001, 98(4):2053 2058.

21. Papenbrock J, Pfndel E, Mock H, Grimm B: Decreased and increased expression of the subunit CHL I diminishes $\mathrm{Mg}$ chelatase activity and reduces chlorophyll synthesis in transgenic tobacco plants. Plant J 2000 22(2):155 164

22. Zhou K, Ren Y, LV J, Wang Y, Liu F, Zhou F, Zhao S, Chen S, Peng C, Zhang X, Guo X, Cheng Z, Wang J, Wu F, Jiang L, Wan J: Young leaf chlorosis 1, a chloroplast-localized gene required for chlorophyll and lutein accumulation during early leaf development in rice. Planta 2013, 237(1):279 292.

23. Zhou Y, Gong Z, Yang Z, Yuan Y, Zhu J, Wang M, Yuan F, Wu S, Wang Z, Yi C, Xu T, Ryon M, Gu M, Liang G: Mutation of the light-induced yellow leaf 1 gene, which encodes a geranylgeranyl reductase, affects chlorophyll biosynthesis and light sensitivity in rice. PLOS One 2013, 8(9):e75299.

24. Wang $L$, Wang $X$, Yue $C$, Cao $H$, Zhou Y, Yang Y: Development of a $44 \mathrm{~K}$ custom oligo microarray using 454 pyrosequencing data for large-scale gene expression analysis of the Camellia sinensis. Sci Hortic 2014, 174:133 141.

25. Arnon D: Copper enzymes in isolated chloroplasts: polyphenoloxidase in beta vulgaris. Plant Physiol 1949, 24:1 15.

26. Wang $X$, Chen L, Ma C, Yao M, Yang Y: Genotypic variation of beta-carotene and lutein contents in tea germplasms, Camellia sinensis (L.) O. Kuntze. J Food Compos Anal 2010, 23(1):9 14

27. Peng $L$, Song $X$, Shi $X$, Li J, Ye C: An improved HPLC method for simultaneous determination of phenolic compounds, purine alkaloids and theanine in Camellia species. J Food Compos Anal 2008, 21(7):559 563.

28. Kerio LC, Wachira FN, Wanyoko JK, Rotich MK: Characterization of anthocyanins in Kenyan teas: Extraction and identification. Food Chem 2012, 131(1):31 38

29. Chang S, Puryear J, Cairney J: A simple and efficient method for isolating RNA from pine trees. Plant Molecular Biology Report 1993, 11(2):113 116

30. Livaka KJ, Schmittgen TD: Analysis of Relative Gene Expression Data Using Real-Time Quantitative PCR and the $2^{-\Delta \Delta C T}$ Method. Methods 2001 25(4):402 408 
31. Shao WF: Pigments in Tea. In Tea Biochemistry. Chapter 1. 3rd edition. Edited by Wan XC. Chinese Agricultural Press; 2003:21 28.

32. Liang Y, Lu J, Shang S: Effect of gibberellins on chemical composition and quality of tea ( Camellia sinensis L.). J Sci Food Agric 1996, 72(4):411 414

33. Yokogoshi H, Kato Y, Sagesaka YM, Takihara-Matsuura T, Kakuda T, Takeuchi N: Reduction effect of theanine on blood pressure and brain 5-hydroxyindoles in spontaneously hypertensive rats. Biosci Biotechnol Biochem 1995, 59(4):615 618.

34. Juneja LR, Chu D, Okubo T, Nagato Y, Yokogoshi H: L-theanine a unique amino acid of green tea and its relaxation effect in humans. Trends Food Sci Technol 1999, 10(6 7):199 204

35. Kakuda T: Neuroprotective effects of the green tea components theanine and catechins. Biol Pharm Bull 2002, 25:1513 1518.

36. Song C, Jung J, Oh J, Kim K: Effects of theanine on the release of brain alpha-wave in adult males. Korean J Nutr 2003, 36(9):918 923.

37. Cooper R: Green tea and theanine: health benefits. Int J Food Sci Nutr 2012, 63(S1):90 97.

38. Koes R, Verweij W, Quattrocchio F: Flavonoids: a colorful model for the regulation and evolution of biochemical pathways. Trends Plant Sci 2005 10(5):236 242

39. Higdon JV, Frei B: Tea catechins and polyphenols: health effects, metabolism, and antioxidant functions. Crit Rev Food Sci Nutr 2003, 43(1):89 143.

40. Deng W, Ogita S, Ashihara H: Ethylamine content and theanine biosynthesis in different organs of Camellia sinensis seedlings. Z Naturforsch 2009, 64c:387 390.

41. Shi C, Yang H, Wei C, Yu O, Zhang Z, Jiang C, Sun J, Li Y, Chen Q, Xia T, Wan $X$ : Deep sequencing of the Camellia sinensis transcriptome revealed candidate genes for major metabolic pathways of tea-specific compounds. BMC Genomics 2011, 12(1):131.

42. Kidron H, Repo S, Johnson MS, Salminen TA: Functional classification of amino acid decarboxylases from the alanine racemase structural family by phylogenetic studies. Mol Biol Evol 2007, 24(1):79 89.

43. Feng L, Gao M, Hou R, Hu X, Zhang L, Wan X, Wei S: Determination of quality constituents in the young leaves of albino tea cultivars. Food Chem 2014, 155:98 104.

44. Howitt CA, Pogson BJ: Carotenoid accumulation and function in seeds and non-green tissues. Plant Cell Environ 2006, 29(3):435 445.

45. Li L, Yuan H: Chromoplast biogenesis and carotenoid accumulation. Arch Biochem Biophys 2013, 539:102 109.

46. Cazzonelli $\mathrm{Cl}$, Pogson BJ: Source to sink: regulation of carotenoid biosynthesis in plants. Trends Plant Sci 2010, 15(5):266 274

47. Anderson IC, Robertson DS: Role of carotenoids in protecting chlorophyll from photodestruction. Plant Physiol 1960, 35:531 534

48. Ma C, Chen L, Wang X, Jin J, Ma J, Yao M, Wang Z: Differential expression analysis of different albescent stages of Anji Baicha (Camellia sinensis (L.) O. Kuntze) using cDNA microarray. Sci Hortic 2012, 148:246 254.

49. Papenbrock J, Mock H, Tanaka R, Kruse E, Grimm B: Role of magnesium chelatase activity in the early steps of the tetrapyrrole biosynthetic pathway. Plant Physiol 2000, 122(4):1161 1170.

50. Espineda CE, Linford AS, Devine D, Brusslan JA: The AtCAO gene, encoding chlorophyll a oxygenase, is required for chlorophyll b synthesis in Arabidopsis thaliana. Proc Natl Acad Sci 1999, 96(18):10507 10511.

51. Besseau S, Hoffmann L, Geoffroy P, Lapierre C, Pollet B, Legrand M: Flavonoid accumulation in Arabidopsis repressed in lignin synthesis affects auxin transport and plant growth. Plant Cell 2007, 19(1):148 162.

52. Xiong L, Li J, Li Y, Yuan L, Liu S, Huang JA, Liu Z: Dynamic changes in catechin levels and catechin biosynthesis-related gene expression in albino tea plants (Camellia sinensis L.). Plant Physiol Biochem 2013, 71:132 143.

53. Winkel-Shirley B: Flavonoid biosynthesis. A colorful model for genetics, biochemistry, cell biology, and biotechnology. Plant Physiol 2001, 126(2):485 493.

doi:10.1186/s12870-014-0352-x

Cite this article as: Wang et al:: Biochemical and transcriptome analyses of a novel chlorophyll-deficient chlorina tea plant cultivar. BMC Plant Biology 2014 14:352.

\section{Submit your next manuscript to BioMed Central and take full advantage of:}

$\otimes$ Convenient online submission

$\nabla$ Thorough peer review

$\otimes$ No space constraints or color $\bowtie$ gure charges

$\nabla$ Immediate publication on acceptance

Q Inclusion in PubMed, CAS, Scopus and Google Scholar

$\otimes$ Research which is freely available for redistribution

Submit your manuscript at www.biomedcentral.com/submit
C Biomed Central 